\title{
Edge Detection in Polarimetric SAR Image based on Bandelet Transform
}

\author{
Roy Thankachan \\ National Institute of Technology \\ Calicut, Kerala \\ India
}

\author{
Sethunadh R. \\ Vikram Sarabhai Space Centre \\ Trivandrum, Kerala \\ India
}

\author{
Ameer P. M. \\ National Institute of Technology \\ Calicut, Kerala \\ India
}

\begin{abstract}
Polarimetric Synthetic Aperture Radar is being used widely in order to extract the features of target image including civilian and military applications. Edge detection in PolSAR images is highly challenging task due to the occurrence of speckle noise in these images although widely used for the strategic applications. This paper presents a multi-resolution edge detection method for PolSAR images using the bandelet transform. Bandelet transform can provide flexible multiscale and directional decomposition of images including SAR images. Edge enhancement of the input image is done after decomposing it using bandelet transform and the resultant bandelet coefficients are modified through the maximisation of the polarimetric contrast between the adjacent subbands using Lagrangian methods. Taking advantage of the directional features of bandelet transform an algorithm is developed for retaining the geometrical features of images such as edges, boundaries etc present in SAR images while ensuring effective speckle noise removal. Here the geometrical features in images are enhanced in the bandelet domain by fusing the different directional subband coefficients at different scales. The performance of this method is verified using real PolSAR images. The result shows that proposed scheme eliminates speckle noise and retrieved edges are continuous and complete.
\end{abstract}

\section{Keywords}

PolSAR, bandelet, speckle, directional subbands

\section{INTRODUCTION}

Synthetic Aperture Radar (SAR) based natural imaging is very widely used in remote sensing in order to gain the weather and daylight independence. Polarimetric SAR technique outperforms conventional SAR technique in feature extraction of natural images [1]. Polarimetric SAR data has proven useful for remote sensing applications such as hydrology, agriculture, oceanography, forestry, disaster monitoring, and military applications.

Edge detection is critical for various synthetic aperture radar applications such as image segmentation, detection of features like roads, coastline, crop area, forest cover and so on. But speckle noise is an issue which plagues polarimetric SAR images and is worsened by its multiplicative nature which induces high probability of false alarm. Sliding window techniques using the ratio of the averages are the norm when it comes to conventional edge detectors [2], [3]. These detectors are simple to implement, however, because of their dependence on window size being selected, they are not immune to noise. Though multiscale analysis tools have been developed for edge detection which could counter many of these issues [4]-[7]. But when it comes to fully polarimetric SAR images, the research efforts on this front have found to be lacking. PolSAR could improve the efficiency of edge detection as compared to a single channel SAR [8],[9]. Majority of the edge detection techniques, when it comes to
PolSAR images are primarily based on Roy's largest eigen value method or Wishart likelihood-ratio test (Wishart-LRT) [9]. Because of the usage of windows, there is a tradeoff between edge locating accuracy and speckle suppression. Further transform based techniques have became popular. A two-scale line detection method was proposed in [7] using curvelet transform. Nonsubsampled contourlet transform (NSCT) based multiscale edge detection method was proposed in [10],[11] and object detection based on bandelet transform dealt in [12] where bandelet applied on the spatial domain. Enhanced edge detector using span driven adaptive window put forwarded in [13]. However it is more or less spatial technique with heterogeneous area adaptation. These methods have reduced the incidence of speckle noise as compared to traditional techniques. In this paper, we propose a multiscale detection based on bandelet transform technique and GCV thresholding. Edge enhancement of each element of the covariance matrix is done at different levels through bandelet domain and fused together for final edge image.

The rest of the paper is organized as follows. Section 2 describes the conceptual review of PolSAR data representation [14],[15], bandelet transform fundamentals and it advantages over wavelet transforms and GCV thresholding employed for the bandelet coefficient shrinkage for the speckle removal [16]. Section III describes the proposed method of edge detection. Section IV details the results of proposed method using actual polarimetric data in comparison with the existing transform based methods. Comparison is done based on a handful of performance indices [17]-[21] expressed both in tabular form and spider plot.

\section{POLSAR DATA REPRESENTATION}

PolSAR images are generated through the coherent interference processes between four correlated channels of $\mathrm{HH}, \mathrm{HV}, \mathrm{VH}$, and $\mathrm{VV}$ where $\mathrm{H}$ and $\mathrm{V}$ are the horizontally and vertically polarized waves of the incident and received signal. For a reciprocal system, $2 \times 2$ complex scattering matrix can be represented by 'S', where

$\mathrm{S}=\left[\begin{array}{ll}S_{h h} & S_{h v} \\ S_{v h} & S_{v v}\end{array}\right]$

where $S_{x y}$ be the complex scattering coefficient in which $x$ and $y$ represents the transmitting and receiving polarizations respectively. Scattering matrix completely describes the behaviour of deterministic scatterers but fails to characterize distributed scatterers where resultant image within the resolution of pixel consists of large number of randomly distributed independent scatters. Hence the coherent superposition of independent scatterers to the resulting scattering matrix which will be formalized by $3 \times 3$ Coherency or covariance matrix $(\mathrm{CM}), \mathrm{C}_{3}$ which is given by 
$\mathrm{C}_{3}=\left[\begin{array}{ccc}\left|S_{h h}\right|^{2} & \sqrt{2} S_{h h} S_{h v}{ }^{*} & S_{h h} S_{v v}{ }^{*} \\ \sqrt{2} S_{h v} S_{h h}{ }^{*} & 2\left|S_{h v}\right|^{2} & \sqrt{2} S_{h v} S_{v v}{ }^{*} \\ S_{v v} S_{h h}{ }^{*} & \sqrt{2} S_{v v} S_{h v}{ }^{*} & \left|S_{v v}\right|^{2}\end{array}\right]$

where $*$ indicates complex conjugates.

Covariance matrix completely characterizes the PolSAR images. Even though Polarimetric SAR imaging having several advantages over optical imaging, the major challenges are low signal to noise ratio, involvement of phase information and complex numbers. Coherent imaging process of PolSAR ends up in multiplicative noise called speckle which significantly reduces PolSAR image quality. Speckle is caused by both the additive and deductive interference of reflected waves by different elementary scatterers.

By using statistical models, speckle noise are characterized with the help of random variables [1][14][15]. The uninvited effects of speckle in a PolSAR image can be filtered out during the pre-processing step of any image applications. This can be done either in the spatial domain or in the transform domain. Spatial domain filtering are basically windows based smoothing or filtering such as median, average etc which results in a smoothing and blurred appearance even though it reduces the speckle noise. The evident drawback of such an approach is the loss in spatial resolution. In [1] it is proposed that all the elements of $\mathrm{CM}$ should be filtered identically to preserve the polarimetric properties and to avoid crosstalk between the polarization channels.

Transform domain based filtering involves applying of suitable threshold values to transform coefficients for effective noise removal. Significant quantity of information is enclosed in a small number of the transform coefficients due to the energy compaction property of transform representation. Even though these schemes involves intricate steps, it is an effective noise removal method and hence used extensively.

\section{BANDELET TRANSFORM}

Geometric regularity of image structures cannot be represented effectively by separable orthogonal basis such as Wavelet, Cosine or Fourier transforms. Edges with sharp transitions are very difficult to represent in an image Bandelet transforms integrating the geometric regularity in an image representation [22]. Bandelet transform which is an orthonormal basis and can be interpreted as an warped wavelet basis that is adapted to natural geometric features of images. Wherever the regularity of image exists, the elongated orthogonal vectors are formed in the same direction. The bandelet transform consists of bandeletization procedure which extracts the geometric image regularity. Even though first generation bandelet transform is non orthogonal in nature, it introduces boundary artifacts due to warping. Second generation bandelet transform [23] was introduced to take care of the boundary artifacts. This involves 2-D wavelet transform and subsequent bandeletization operation

The Bandelet Transform initially subdivide the image into smaller blocks and these are successively subdivided until there is only one contour or curve inside each square area. Geometric flow in each of the sub-squares is found out along all possible directions up to $2 n^{2}$ where $n$ is the width of the sub-squares in pixels. The given direction of contour is projected along all the feasible directions. For any particular direction $\mathrm{d}$, a $1 \mathrm{D}$ discrete signal, $\mathrm{f}_{\mathrm{d}}$ is made by projecting each point to the given direction and then subjected to $1 \mathrm{D}$ discrete wavelet transform. Then the best possible direction is found out by the least difference for a specified threshold, $\mathrm{T}$. The bandelet transform finds the best possible direction for a particular user-defined threshold $\mathrm{T}$ by Lagrangian cost function $\mathcal{L}\left(\mathrm{f}_{\mathrm{d}}, \mathrm{R}\right)$ minimisation process where Lagrangian cost function is given by,

$\mathcal{L}\left(\mathrm{f}_{\mathrm{d}}, \mathrm{R}\right)=\left\|\mathrm{f}_{\mathrm{d}}-\mathrm{f}_{\mathrm{dR}}\right\|^{2}+\lambda \mathrm{T}^{2}(\mathrm{R})$

where $f_{d R}$ is the signal reconstructed from the $1 \mathrm{D}$ inverse wavelet transform coefficients. $\mathrm{R}$ number of bits which is used to represent the geometric parameter and quantized coefficients and $\lambda$ is Lagrangian multiplier. The optimum directional approximations for all the dyadic squares are found out by repeating the same procedure by minimizing the Lagrangian cost function and the corresponding quad tree can be built.

Bandelet transform having advantage of retaining the geometric characteristics of original image to the minutest level. The main drawback of wavelet transform lies where the horizontal, vertical and diagonal features are represented prominently. However other directional transforms like bandelet, the directional features are extracted and represented.

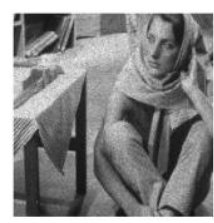

(a)

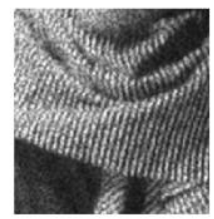

(d)

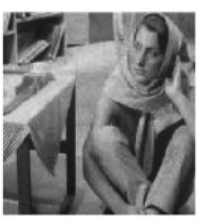

(b)

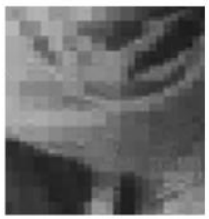

(e)

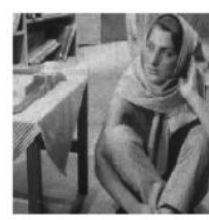

(c)

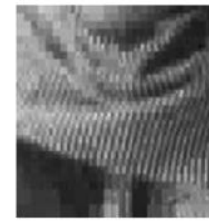

(f)
Fig.1. (a) Barbara image with speckle noise variance $=0.05$

(b) Denoising using wavelet thresholding PSNR $=\mathbf{2 4 . 4 6 \mathrm { dB }}$ (c) Denoising using bandelet thresholding PSNR = 26.82 dB (d) Heterogenous area of barbara (e) Wavelet based denoising PSNR = 19.12dB (f) Bandelet based denoising PSNR $=21.02 \mathrm{~dB}$

The effectiveness of the directional feature retention can be evaluated by using the simple thresholding algorithm after computing the transform coefficients. Barbara image shown in Fig.1 with speckle noise variance 0.05 is despeckled by applying hard threshold on bandelet coefficients and reconstructing back using inverse operations and is compared with wavelet based thresholding.

Bandelet based denoising outperforms the other method based on wavelet thresholding, both qualitatively and quantitatively. Mean Square Error and PSNR comparison of the both the images shown in Table.1 indicates the better speckle suppression and feature retention for bandelet based thresholding. 
Table.1. Performance indices for barbara image with different noise variance

\begin{tabular}{|c|c|c|c|c|}
\hline \multirow{2}{*}{$\begin{array}{c}\text { Noise } \\
\text { Variance }\end{array}$} & \multicolumn{2}{|c|}{ Wavelet } & \multicolumn{2}{c|}{ Bandelet } \\
\cline { 2 - 5 } & MSE & PSNR & MSE & PSNR \\
\hline $\mathbf{0 . 0 5}$ & 1.2 & 23.2 & 0.7 & 28.0 \\
\hline $\mathbf{0 . 1}$ & 3.8 & 21.7 & 1.6 & 25.9 \\
\hline $\mathbf{0 . 2}$ & 5.2 & 19.7 & 2.9 & 23.6 \\
\hline
\end{tabular}

\section{GCV THRESHOLD}

Selection of the optimal threshold is the major challenge in any transform based filtering scheme. A higher threshold will result in a loss of significant features in the image, on the other side a smaller threshold will not effectively remove speckle. Generalized Cross Validation (GCV) [16] method can be adopted to select an optimal threshold without knowing the noise variance. This method is more suited to PolSAR images where target image noise variance not known. In this method, the optimal threshold $\tau$ is defined as the smallest value of the GCV function. In transform domain GCV function for a particular subband $d$ as a function of threshold $\tau$, is defined as

$$
\operatorname{GCV}_{\mathrm{d}}(\tau)=\frac{\frac{1}{\mathrm{~N}_{\mathrm{d}}}\left\|\mathrm{Y}_{\mathrm{d}}-\mathrm{Y}_{\mathrm{d}, \tau}\right\|^{2}}{\left[\frac{\mathrm{N}_{\mathrm{d} 0}}{\mathrm{~N}_{\mathrm{d}}}\right]^{2}}
$$

where $N_{d}$ is number of bandelet coefficients in $d$ th subband, $Y_{d}$ represents bandelet coefficients with noise, $Y_{d} \tau$ is resultant coefficients with the applied threshold and $\mathrm{N}_{\mathrm{d} 0}$ represents the number of coefficients below the threshold. Generalized cross validation for bandelet shrinking is done on the absolute value of the bandelet coefficients.

Motivated by the geometric feature retention capability of the bandelet transform, we propose a new method for despeckling the PolSAR in transform domain and further edge enhancement \& detection.

\section{PROPOSED METHOD}

In this section, the bandelet transform is modified to suit for PolSAR image processing and the stepwise details of the proposed bandelet transform based edge detection method is explained.

Multilevel images are generated from the input covariance image by multiplying with Gaussian mask. Here a 3 level process is suggested. Maximisation of the polarimetric contrast of the input image is done at three levels using the optimisation of Ratio Laplacian Pyramid. Ratio Laplacian Pyramid for fully PolSAR images is defined by a ratio of two Hermitian forms [22], as

$$
R_{k}(m, n)=\frac{F^{H} C_{k-1}(\mathrm{~m}, \mathrm{n}) \mathrm{F}}{F^{H} C_{k}(\mathrm{~m}, \mathrm{n}) \mathrm{F}}
$$

where $F$ is a vector to be optimized, $C_{k}$ represents the polarimetric covariance matrix data in the $\mathrm{k}^{\text {th }}$ layer, which is generated by convolution of $\mathrm{C}_{\mathrm{k}-1}$ with a Gaussian mask, and $\mathrm{C}_{0}$ is the original polarimetric covariance matrix data. Note that $\mathrm{C}_{\mathrm{k}}$ is a low pass form of $\mathrm{C}_{\mathrm{k}-1}$. Therefore the ratio $\mathrm{R}_{\mathrm{k}}$ can be considered as the polarimetric contrast between adjacent layers, and a higher contrast is conducive to extracting edges. The maximization of $\mathrm{R}_{\mathrm{k}}(\mathrm{m}, \mathrm{n})$, in (5) is a generalized Rayleigh quotient problem i.e. when $F$ is the largest eigenvector of $C_{k}(m, n)^{-1} C_{k-1}(m, n), R_{k}(m, n)$ achieves its maximum[13], i.e. the maximum eigen value of $\mathrm{C}_{\mathrm{k}}(\mathrm{m}, \mathrm{n})^{-1} \mathrm{C}_{\mathrm{k}-1}(\mathrm{~m}, \mathrm{n})$. Considering that PolSAR data often have a wide dynamic range, taking the logarithm of the decomposition result can benefit the variable histogram equilibrium. Therefore, the contrast enhanced image is

$\operatorname{Max} \mathrm{R}_{\mathrm{k}}(\mathrm{m}, \mathrm{n})=\log \left(\max \left\{\operatorname{eig}\left[\mathrm{C}_{\mathrm{k}}(\mathrm{m}, \mathrm{n})^{-1} \mathrm{C}_{\mathrm{k}-1}(\mathrm{~m}, \mathrm{n})\right]\right\}\right)$

According to the above definition, the modified equation can be treated as a reasonable depiction of the geometric information in PolSAR image. Contrast enhanced image is despeckled using bandelet transform and GCV thresholding.

The speckle noise which is granular in nature possessing the multiplicative characteristics. So in order to make any filtering scheme effective, this must be converted to an additive noise. For that purpose a logarithmic transformation is applied as a pre-processing step to the PolSAR image. Then, the transform coefficients are found out by using second generation bandelet transform . Noise energy will be distributed all over the coefficients at the same time the major image geometrical features are kept in a few bandelet coefficients [12]. So the reflectance of noise on the large valued signal coefficients will be negligible. Then soft thresholding operation is done in order to remove the high frequency noise components. Inverse bandelet transform is applied to the resultant soft thresholded bandelet coefficients to reconstruct back the edge enhanced and speckle-reduced image. Selection of the optimal threshold is the major challenge which is a tradeoff between the noise level and signal geometric features. A higher threshold can result in loss of major geometric features while a smaller threshold will retain the noise components in an image. Generalized Cross Validation (GCV) scheme is used here to find out an optimal threshold.

Then, absolute value of each of these levels is found out by normalizing with their maximum value. Identical to the detection of edges using wavelet transform, edge intensive area at each scale is determined by finding out the modulus maxima of all the multidirectional subband bandelet coefficients. The direction of the subband that has the maximum modulus represents the edge orientation 


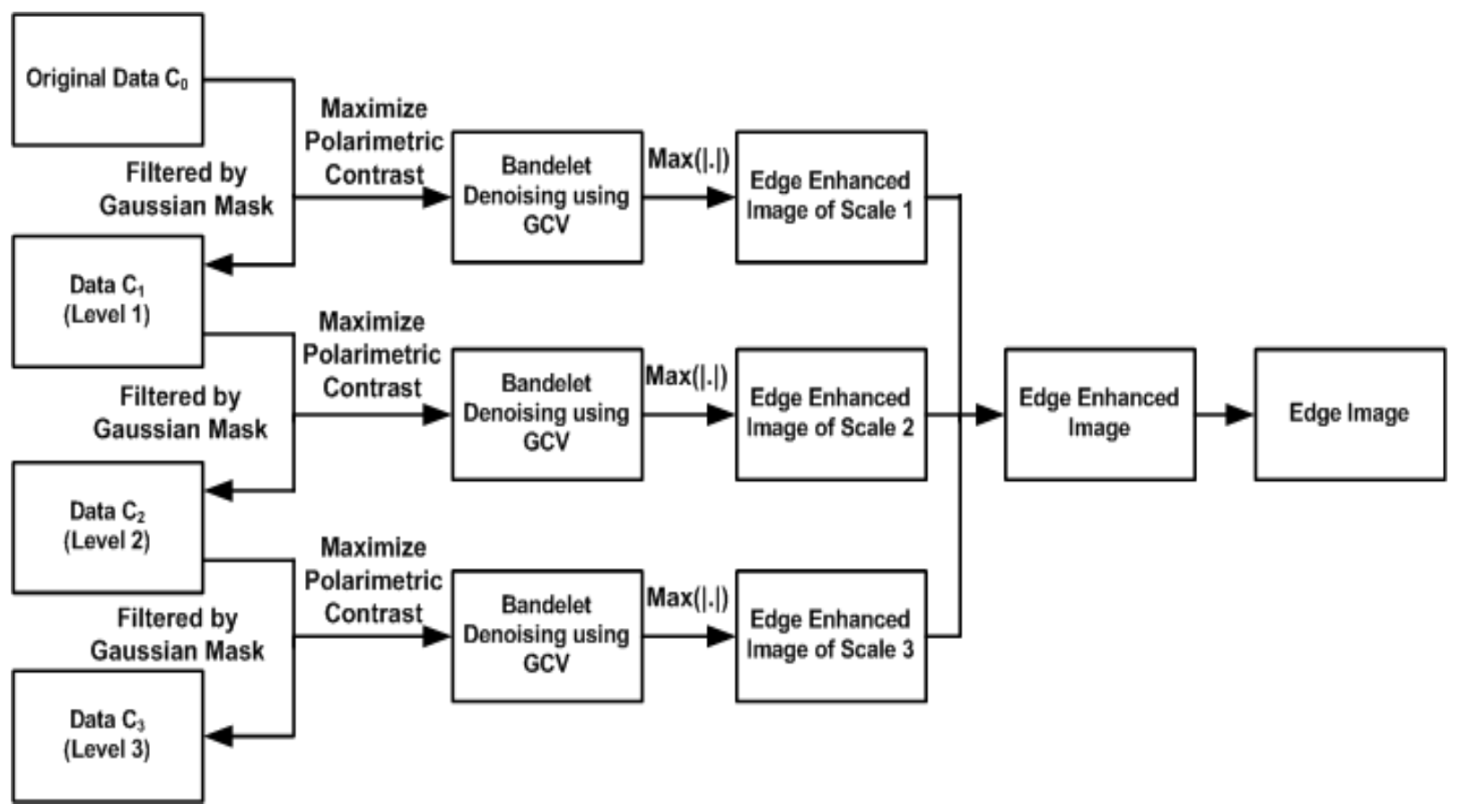

Fig.2. Implementation methodology of the proposed edge detection method

Then, point wise multiplication is done to combine these subband maximum values, which means that after this process, each pixel output will be the product of the maximum values obtained at different scales. The block diagram of the proposed method is shown in Fig.2.

The fine scale image could obtain rich edge details and accurate edge locations, while the coarse scale image can detect stable edges and has a lower false alarm rate. A combination of several scales can take the advantages in both preserving real edges and reducing false alarms.

\section{EXPERIMENTAL RESULTS}

Real PolSAR images acquired by both airborne and space borne systems were used for evaluating the effectiveness of the proposed scheme. AIRSAR Flevoland (NL), STK-MLC format $750 \times 1024$ image and EMISAR Foulum Denmark $1100 \times 1772$ image are used to evaluate the performance of the proposed method with the other state of art methods. A brief description of the quantitative performance indices used are given below.

Equivalent Number of Looks (ENL): This is one of the important index in coherent systems like PolSAR filtering processing which depicts the efficiency in removing speckle noise of image over homogeneous areas. ENL is given by,

$$
\begin{aligned}
& \mathrm{ENL}=\mathrm{A}\left(\frac{\mu^{2}}{\sigma^{2}}\right) \\
& \text { (6) } \sigma^{2}=
\end{aligned}
$$

variance, $\mu=$ mean

Higher value of ENL corresponds to a better filter performance. However absolute value of ENL largely depends on the homogeneity of the area selected, image size etc. Judicious selection of the homogenous area within the image is very much important in parametric evaluation and performance assessment. Also, if the image area is larger, the ENL value will be higher and vice versa. ENL is a simple performance metric to measure the similarity among the specked input image and the denoised output image of same size.
Speckle Suppression Index (SSI): It is the ratio of coefficient of variations of despeckled PolSAR image to the original PolSAR image and is given as

$\mathrm{SSI}=\frac{\sqrt{\operatorname{var}(\mathrm{Z})}}{\operatorname{mean}(\mathrm{Z})} \frac{\operatorname{mean}(\mathrm{Y})}{\sqrt{\operatorname{var}(\mathrm{Y})}}$

where $\mathrm{Z}=$ despeckled Image, $\mathrm{Y}=$ Image with speckle noise

SSI value should be less than 1 . Lower the value means higher the speckle reduction. Ideal value of SSI is zero.

Mean Preservation Index (MPI): This is the index which reveals the over smoothing characteristics of filters with respect to the original image. Mean of the original image is to be preserved even after speckle filtering. To quantify the mean preservation filter capability, a selective comparison of the sample mean of the original image to the filtered denoised image is obtained for homogeneous area. Mean Preservation Index (MPI) is defined as

Mean Preservation Index,

$\mathrm{MPI}=\left|\frac{\operatorname{mean}(\mathrm{Y})-\operatorname{mean}(\mathrm{Z})}{\operatorname{mean}(\mathrm{Y})}\right|$

where $\mathrm{Z}=$ despeckled Image, $\mathrm{Y}=$ Image with speckle noise

Lower value of MPI indicates superior filter performance with respect to mean preservation and speckle noise reduction, independent of the absolute mean value. Since it is a normalized parameter MPI is a good indicator of the mean preservation capability of a filter independent of the actual image content.

Edge Save Index or Edge Preserve Index (ESI or EPI): Edge Save Index corresponds to the edge preservation capability of SAR image of the despeckling technique. ESI is a metric that quantifies the edge save ability of an edge detection algorithm in Horizontal and Vertical directions, represented as $\mathrm{ESI}_{\mathrm{H}}$ and ESI $_{\mathrm{V}}$ respectively. It is computed as

Edge Save Index Horizontal, 
$\operatorname{ESI}_{H}=\frac{\sum_{i=1}^{m} \sum_{j=1}^{n-1}\left|\hat{Y}_{i, j+1}-\hat{Y}_{i, j}\right|}{\sum_{i=1}^{m} \sum_{j=1}^{n-1}\left|Y_{i, j+1}-Y_{i, j}\right|}$

Edge Save Index Vertical,

$\operatorname{ESI}_{V}=\frac{\sum_{i=1}^{\mathrm{n}} \sum_{\mathrm{j}=1}^{\mathrm{m}-1}\left|\hat{\mathrm{Y}}_{\mathrm{i}+1, j}-\hat{\mathrm{Y}}_{\mathrm{i}, \mathrm{j}}\right|}{\sum_{\mathrm{i}=1}^{\mathrm{n}} \sum_{\mathrm{j}=1}^{\mathrm{m}-1}\left|\mathrm{Y}_{\mathrm{i}+1, \mathrm{j}}-\mathrm{Y}_{\mathrm{i}, \mathrm{j}}\right|}$

where $\hat{Y}_{i, j}$ is despeckled SAR image, $Y_{i, j}$ is original SAR image, $m$ is the number of row in SAR image and $n$ is the number of columns in SAR. If this value is more, the preservation of the edges of the image is more. It should be high. Maximum value of $\mathrm{ESI}_{\mathrm{H}}$ or $\mathrm{ESI}_{\mathrm{V}}$ is 1 . A better visible image is always having reasonable good ESI value.

Mean Preservation Speckle Suppression Index (MPSSI): It is a better normalized index with respect to SMPI and is better for a comparison of various filters on different images. MPSSI is defined as

Mean Preservation and Speckle Suppression Index,

MPSSI $=\left|1-\frac{\operatorname{mean}(\mathrm{Z})}{\operatorname{mean}(\mathrm{Y})}\right| * \frac{\sqrt{\operatorname{var}(\mathrm{Z})}}{\sqrt{\operatorname{var}(\mathrm{Y})}}$

$\mathrm{Z}=$ Despeckled Image, $\mathrm{Y}=$ Speckled Image

The lower value of MPSSI indicates better performance of the filter in terms of the mean preservation and noise reduction, independently of the actual mean value

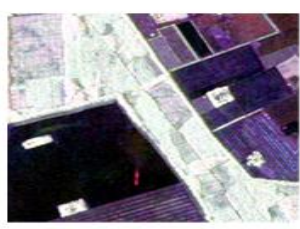

(a)

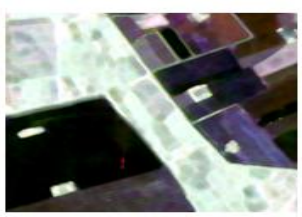

(c)

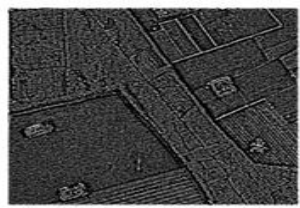

(e)

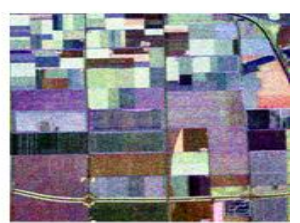

(b)

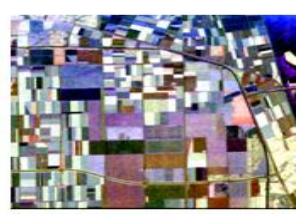

(d)

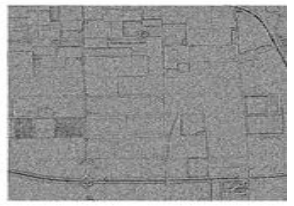

(f)
Fig.4. (a) Pauli RGB image of AIRSAR Flevoland (b) EMISAR L Band Foulum Denmark (c) \& (d) Despeckled image using Bandelet transform (e) \& (f) edge enhanced image of $(\mathbf{a}) \&(b)$

The most key feature of the proposed method is that the elements of the covariance matrix are processed according to heterogeneous content of the image. Quantitative and qualitative evaluation of both airborne and space borne PolSAR data establishes the proposed method does not result any degradation in polarimetric features and improves the estimation of edges in the image.

Fig.4 shows the original Pauli RGB image, despeckled image and its edge enhanced image using the proposed procedure of AIRSAR Flevoland and EMISAR Foulum Denmark and Fig.5 (a),(b) \& (c) depicts the edges detected in the enhanced image of Foulum Denmark using Shearlet based thresholding [8], Non subsampled Contourlet transform [22] and proposed edge detection method. From the figures it is well evident that the proposed method outperforms the other methods in terms of detecting the true edges avoiding the false edge representations.

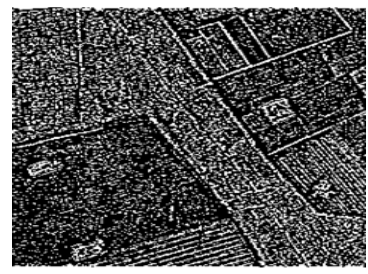

(a)

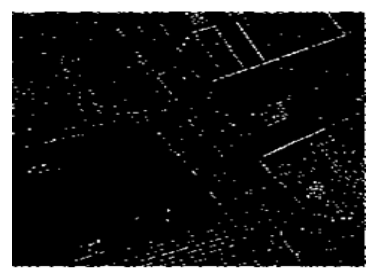

(b)

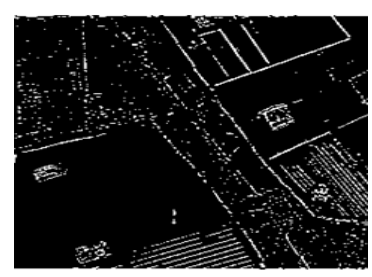

(c)

Fig.5. Edge detected images of EMISAR L Band Foulum Denmark (a) Shearlet based thresholding (b) NSCT based method (c) Proposed method

Table. 2 contains the performance indices of the despeckled image using Shearlet, NSCT and Bandelet. There is a significant increase in SSI, ESI $\mathrm{H}_{\mathrm{H}}$ and $\mathrm{ESI}_{\mathrm{V}}$ for the bandelet method. Similarly MPI and MPSSI also showed improvement. However better ENL obtained for NSCT method. This could be due to the averaging of NSCT coefficients.

Table.2. Performance indices of edge enhanced image of EMISAR L Band Foulum Denmark

\begin{tabular}{|c|c|c|c|c|c|c|}
\hline Scheme & ENL & SSI & MPI & MPSSI & ESI $_{\mathrm{H}}$ & ESI $_{\mathrm{V}}$ \\
\hline Shearlet & 24.2 & 0.42 & 0.65 & 0.014 & 0.872 & 0.824 \\
\hline NSCT & 25.6 & 0.33 & 0.63 & 0.025 & 0.877 & 0.825 \\
\hline $\begin{array}{c}\text { Proposed } \\
\text { Method }\end{array}$ & 26.1 & 0.31 & 0.59 & 0.009 & 0.889 & 0.839 \\
\hline
\end{tabular}




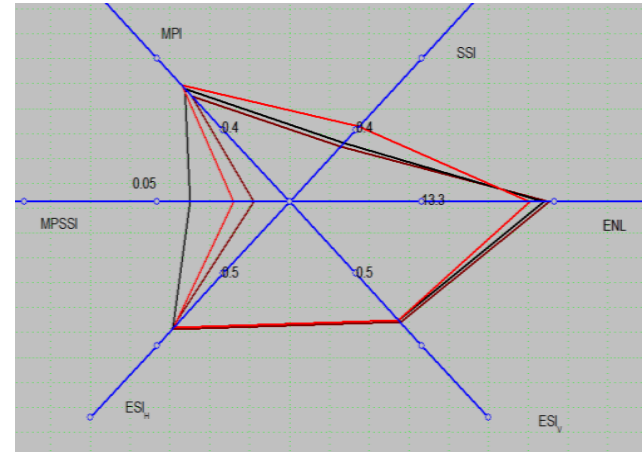

Fig.6. Spider plot of performance indices of different edge detection schemes - Shearlet (Red), NSCT (Black) and proposed method(Brown).

\section{CONCLUSION}

A novel method for edge detection in Polarimetric SAR image using bandelet transform is presented in this paper. The edge detection is done at different subbands at different levels using bandelet transform to avoid false detection and robust estimation of edge characteristics. GCV threshold is effective to filter out the speckle noise and proves optimal threshold for despeckling. Effectiveness of the algorithm is verified using the real PolSAR images in terms of both subjective visual quality and edge details preservation.

\section{REFERENCES}

[1] J. Sen Lee and E.Pottier, "Overview of Polarimetric Radar Imaging," in Polarimetric Radar Imaging From Basics to Applications, New York: CRC Press., 2013, pp. 2-28.

[2] R. Touzi, A. Lopès, and P. Bousquet, "A statistical and geometrical edge detector for SAR images," IEEE Trans. Geosci. Remote Sens., vol. 26,no. 6, pp. 764-773, Nov. 1988.

[3] C. J. Oliver, D. Blacknell, and R. G. White, "Optimum edge detection in SAR,” Proc. Inst. Elect. Eng., vol. 143, no. 1, pp. 31-40, Feb. 1996.

[4] M. T. Alonso, C. Lopez-Martinez, J. J. Mallorqui, and P. Salembier, "Edge enhancement algorithm based on the wavelet transform for automatic edge detection in SAR images," IEEE Trans. Geosci. Remote Sens.,vol. 49, no. 1, pp. 222-235, Jan. 2011.

[5] G. Y. Zhou, Y. Cui, Y. L. Chen, J. Yang, and H. F. Rashvand, "SAR image edge detection using curvelet transform and Duda operator," Electron. Lett., vol. 46, no. 2, pp. 167-169, Jan. 2009.

[6] S. Yi, D. Labate, G. R. Easley, and H. Krim, "A shearlet approach to edge analysis and detection," IEEE Trans. Image Process., vol. 18, no. 5, pp. 929-941, May 2009.

[7] Q.W. Li, G. Y. Huo, H. Li, G. C. Ma, and A. Y. Shi, "Bionic vision-based synthetic aperture radar image edge detection method in nonsubsampled contourlet transform domain,” IET Radar, Sonar Navigat., vol. 6, no. 6, pp. 526-535, Jul. 2012.

[8] J. Schou, H. Skriver, A. A. Nielsen, and K. Conradsen, "CFAR edge detector for polarimetric SAR images," IEEE Trans. Geosci. Remote Sens., vol. 41, no. 1, pp. 20-32, Jan. 2003.
[9] G. Y. Zhou et al., "Linear feature detection in polarimetric SAR images," IEEE Trans. Geosci. Remote Sens., vol. 49, no. 4, pp. 1453-1463, Apr. 2011.

[10] R. J. Jin, J. J. Yin, W. Zhou, and J. Yang, "Edge detection in polarimetric SAR images based on the nonsubsampled contourlet transform," in Proc.IEEE Radar Con., May 2015, pp.0319-0323

[11] J.Rujin and Junjun Yin, "Improved multiscale edge detection method for polarimetric SAR images", IEEE Trans. Geosci. Remote Sens., vol. 13, no.8,pp. 1104 1108, Aug. 2016

[12] N. Raj, R.Sethunadh and P.R. Aparna," Object detection in SAR image based on bandelet transform", J. Vis. Commun. Image R. vol. 40, pp. 376-383, 2016

[13] W.Wang, D. Xiang et al "Enhanced edge detection for polarimetric SAR images using a directional span-driven adaptive window", Int. Journal of Remote Sensing, 2018

[14] J. Sen Lee and E.Pottier, "Electromagnetic Vector Scattering Operators," in Polarimetric Radar Imaging From Basics to Applications, New York: CRC Press. 2013, pp. 53-72.

[15] J. Sen Lee and E.Pottier, "Polarimetric SAR Speckle Filtering," in Polarimetric Radar Imaging From Basics to Applications, New York: CRC Press., 2013, pp. 143-147.

[16] M.Jansen, M. Malfait and A. Bultheel, "Generalized cross validation for wavelet thresholding", IEEE Trans. Signal Process., vol. 56 no.1, pp. 33-44, 1997

[17] S. Al Zubi, N. Islam and M. Abbod, " Multiresolution analysis using wavelet, ridgelet and curvelet transforms for medical image segmentation," Int. J. of Biomedical Imaging, vol. 2011

[18] Niedermeier, A., E. Romaneessen, and S. Lehner. "Detection of Coastlines in SAR Images Using Wavelet Methods." IEEE Transactions on Geoscience and Remote Sensing vol.38 (5): pp. 2270-2281, 2000

[19] Nascimento, A. D. C., M. M. Horta, A. C. Frery, and R. J. Cintra. "Comparing Edge Detection Methods Based on Stochastic Entropies and Distances for PolSAR Imagery." IEEE Journal of Selected Topics in Applied Earth Observations and Remote Sensing .vol.7 (2), pp. 648-663. 2014

[20] D.Xiang, Y.Ban, W.Wang,T.Tang and Y. Su, "Edge Detector for Polarimetric SAR Images Using SIRV Model and Gauss-Shaped Filter," IEEE Trans. Geosci. Remote Sens., vol.13, no.11 pp. 1661-1665,Nov. 2016.

[21] B. Liu, Z. Zhang, X. Liu, and W. Yu, "Edge extraction for polarimetric SAR images using degenerate filter with weighted maximum likelihood estimation," IEEE Trans. Geosci. Remote Sens., vol. 11, no.12 pp. 2140-2144, Dec. 2014.

[22] E. Le Pennec and S.Mallat, "Sparse geometric image representation with bandelets", IEEE Trans. Image Process., vol. 14, no.4, pp. 423-438, Apr. 2005

[23] G. Peyre and S.Mallat, "Orthogonal bandelet bases for geometric images approximation," Commun. Pure and Appl. Math., vol.000, pp. 0001-0029, 2000 